4-1-2012

\title{
Organic-Vapor-Liquid-Solid Deposition with an Impinging Gas Jet
}

Daniel W. (Daniel Wainwright) Shaw

Western Washington University

Kevin A. (Kevin Anthony) Bufkin

Western Washington University

Alexandr A. Baronov

Brad L. Johnson

Western Washington University

David L. Patrick

Western Washington University, david.patrick@wwu.edu

Follow this and additional works at: https://cedar.wwu.edu/chemistry_facpubs

Part of the Chemistry Commons

\section{Recommended Citation}

Shaw, Daniel W. (Daniel Wainwright); Bufkin, Kevin A. (Kevin Anthony); Baronov, Alexandr A.; Johnson, Brad L.; and Patrick, David L., "Organic-Vapor-Liquid-Solid Deposition with an Impinging Gas Jet" (2012). Chemistry Faculty and Staff Publications. 2.

https://cedar.wwu.edu/chemistry_facpubs/2 


\title{
Organic-vapor-liquid-solid deposition with an impinging gas jet
}

\author{
Daniel W. Shaw, ${ }^{1}$ Kevin Bufkin, ${ }^{1}$ Alexandr A. Baronov, ${ }^{1}$ Brad L. Johnson, ${ }^{2}$ \\ and David L. Patrick ${ }^{1, a)}$ \\ ${ }^{1}$ Department of Chemistry, Western Washington University, 516 High St., Bellingham, \\ Washington 98225, USA \\ ${ }^{2}$ Department of Physics, Western Washington University, 516 High St., Bellingham, Washington 98225, USA
}

(Received 20 October 2011; accepted 2 March 2012; published online 13 April 2012)

\begin{abstract}
A method for rapid, mass-efficient deposition of highly crystalline organic films under near ambient conditions of pressure and temperature is reported based on delivery of an organic precursor via an impinging gas jet to a substrate coated by a thin liquid solvent layer. Films of the organic semiconductor tetracene were deposited by sublimation into a flow of argon carrier gas directed at an indium-tin-oxide/glass substrate coated by a thin layer of bis(2-ethylhexyl)sebecate, and growth was followed in situ with optical microscopy. A fluid dynamics model is applied to account for the gas phase transport and aggregation, and the results compared to experiment. The combination of gas jet delivery with an organic-vapor-liquid-solid growth mechanism leads to larger crystals and lower nucleation densities than on bare surfaces, with markedly different nucleation and growth kinetics. An explanation based on enhanced solution-phase diffusivity and a larger critical nucleus size in the liquid layer is proposed to account for the differences. (C) 2012 American Institute of Physics. [http://dx.doi.org/10.1063/1.3699367]
\end{abstract}

\section{INTRODUCTION}

Thin films of organic molecular crystals (OMCs) have received steadily increasing attention over the last 30 years as a result of their interesting and potentially useful electronic, optical, magnetic, and chemical properties. ${ }^{1}$ The scope of application for these materials is remarkably broad: organic crystalline films have been used in transistors, light emitting diodes, and photovoltaics; ${ }^{2}$ as chemical sensors; ${ }^{3}$ and as conductors, ${ }^{4}$ semiconductors, ${ }^{5}$ superconductors, ${ }^{6}$ and magnetic materials. ${ }^{7}$ While few of the properties required for these applications are currently as well developed in organic systems as in their inorganic counterparts, organic crystalline films do offer other important advantages, such as the potential to be processed using relatively low-cost methods. In organic materials, charge transport and many other properties of interest are governed by both the characteristics of the individual molecules and their spatial arrangement. ${ }^{8}$ Crystal structure, grain size and shape, and crystallographic orientation are all important. ${ }^{9-11}$ It is for this reason that relatively few thin film devices have been produced approaching the same level of performance as single crystals, demonstrating how important it is to grow films of sufficiently high quality, and that careful control over molecular order at nanometer to micron length scales is essential. ${ }^{12-15}$

Many OMC compounds have poor solubility, so crystalline films are often prepared by physical vapor deposition (PVD) or molecular beam epitaxy (OMBE). ${ }^{16}$ However, in addition to their relatively high cost and low throughput, films grown using these techniques often consist of small $(<1 \mu \mathrm{m}$ sized) crystals, and it is relatively difficult to control crystal structure, orientation, or growth habit. ${ }^{17}$ In an effort to address

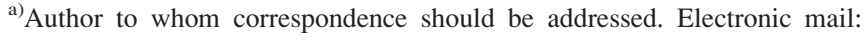
david.patrick@wwu.edu.Fax 360-650-2826.
}

some of these limitations, recently, a hybrid thin film deposition technique combining elements of PVD with solutionphase growth was proposed in which an organic vapor is delivered to a substrate coated by a thin liquid layer. ${ }^{18} \mathrm{~A}$ steady flux of new growth units to the solvent film maintains a supersaturated state leading to crystal nucleation and growth in a liquid environment, thus circumventing the challenge posed by low solubility (Fig. 1). The method is similar to the vapor-liquid-solid (VLS) technique of crystal growth introduced by Wagner and Ellis for growing inorganic crystalline whiskers in a liquid metal alloy droplet, ${ }^{19-22}$ but employs an organic, ${ }^{18,23-25}$ ionic liquid, ${ }^{26}$ or liquid crystalline ${ }^{27}$ solvent combined with an organic precursor. When applied to organic systems, the technique has been called organic-VLS (OVLS).

In the OVLS technique, crystal formation takes place at the gas-liquid or liquid-solid interface, or crystals may form freely floating fully imbedded in the solvent, depending on the wetting properties of the crystal/solvent system. ${ }^{18-27}$ If confined to the liquid layer, quasi 2-dimensional growth takes place, and in the case of liquid crystalline solvents, films with controlled uniaxial in-plane crystallographic orientation have been produced. ${ }^{27}$ Because molecular diffusion occurs more readily in liquids than on bare surfaces, films grown using OVLS tend to develop fewer, but larger crystals than in films of the same compound deposited onto bare surfaces. Crystallization in a liquid may also afford better control over the concentration of incorporated impurities and crystallite morphology through solvent chemical interactions. Particularly for the preparation of OMC films for electronic and optoelectronic applications, where crystallite size, orientation, and purity strongly affect performance, ${ }^{28,29}$ these features of OVLS have the potential to make it a useful alternative to PVD and OMBE. Another advantage of OVLS deposition is that it can in principle be performed under near ambient conditions of pressure and temperature while still 


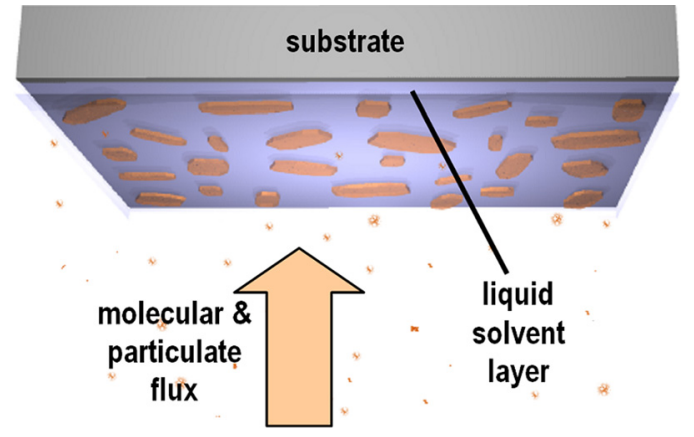

FIG. 1. OVLS combines aspects of vapor-phase deposition with solutionphase growth in a thin solvent layer. The organic precursor is supplied as a vapor in the form of monomers and small particles, saturating the solvent layer and causing crystals to grow, imbedded in the fluid.

producing highly crystalline films. The higher diffusivity in liquids compared to bare surfaces allows the substrate temperature to be kept low, and the tendency of small clusters to dissolve in the liquid permits the use of vapor delivery conditions in which some gas-phase aggregation of the growth precursor can be tolerated. By contrast, during deposition onto a bare surface the existence of small gas-phase aggregates normally tends to produce amorphous films. ${ }^{30-32}$

The OVLS film growth technique was first reported by Voigt and coworkers a few years ago ${ }^{18}$ and so far has only been investigated in a few systems and under a limited range of experimental conditions. ${ }^{19-27}$ To date most studies have employed low or high vacuum conditions with the organic precursor delivered as a monomeric flux from a sublimation source. The reason for the use of low pressure conditions is that, at ambient pressure, vapor molecules approaching the substrate must cross a stagnant diffusive boundary layer adjacent to the interface which has the effect of inhibiting transport to the surface. ${ }^{37}$ The effect can be so large that deposition rates and efficiencies become impractically small. If OVLS deposition is to be performed near ambient conditions, a method of increasing the deposition efficiency by overcoming the hydrodynamic boundary condition is therefore required.

Here it is shown that by delivering vapor carried within an impinging gas jet, relatively rapid deposition rates and high mass deposition efficiencies can be achieved under ambient conditions while still providing for the growth of highly crystalline films with large grain sizes. Through studies of film deposition and growth focusing on the model organic semiconductor tetracene, a fluid dynamics model is applied to account for gas phase transport and aggregation, and the results compared to experiment. The resulting films are observed to display morphologies and growth kinetics which differ significantly from those of similar films deposited onto bare surfaces. Similar results were also found for rubrene and $\alpha$-sexithiophene film, suggesting these findings should be applicable to a broad class of OMC materials.

\section{Experimental methods}

A sketch of the experimental apparatus is shown in Fig. 2. A finely ground powder of an organic semiconductor (tetracene, anthracene, rubrene, or $\alpha$-sexithiophene) was placed in

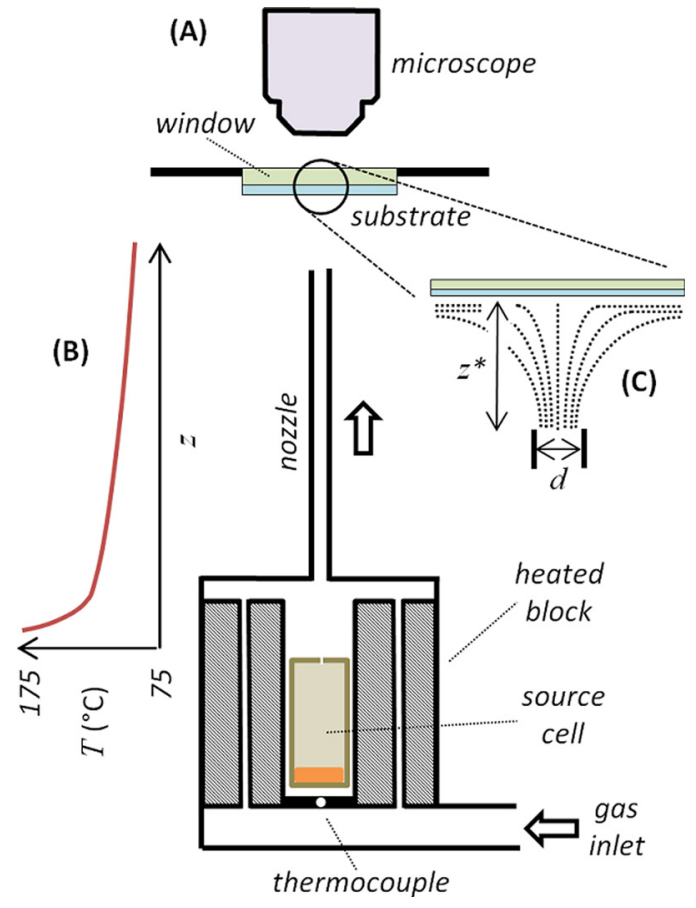

FIG. 2. (a) Schematic of the experimental apparatus. Sublimate exited the heated source cell through a pinhole opening and was carried by a stream of Argon out the nozzle toward the substrate. A shutter between the nozzle and substrate (not shown) was used to control deposition. Film growth was monitored in situ through a transparent window with polarized optical microscopy. (b) The temperature of the gas stream decreased as it flowed through the nozzle toward the sample. (c) The geometry of the impinging jet produces axisymmetric stagnation point flow at the substrate.

an aluminum source cell held in a ceramic heater positioned at the base of an airtight chamber. In the following we focus on results from tetracene films, with a brief discussion of findings from $\alpha$-sexithiophene given at the end. The source cell was sealed with a high temperature Kalrez ${ }^{\circledR}$ o-ring, with vapor allowed to escape through a $340 \mu \mathrm{m}$ diameter hole in the top. The use of a small hole was found to be important for achieving steady deposition rates and reproducible results under the near atmospheric pressure conditions in the chamber. With this configuration the flux was observed to be independent of the mass loading in the source cell, varied approximately exponentially with cell temperature, and was nearly constant in time (over a periods as long as $10 \mathrm{~h}$ ), thus approximating the properties of an effusion cell. The temperature of the cell, which was typically held at $175-200^{\circ} \mathrm{C}$, was measured with a calibrated thermocouple positioned at its base.

Argon carrier gas was introduced through an inlet at the bottom of the ceramic heater and passed through a network of small channels, allowing the gas to be heated close to the same temperature as the source cell. Tetracene vapor issuing from the source cell was swept into the gas flow, exiting through a $7 \mathrm{~cm}$ long circular nozzle whose flow was directed onto the sample. For most experiments a nozzle with an inner diameter of $d=3.81 \mathrm{~mm}$ was used, and the distance between the sample and the end of the nozzle was $z^{*}=13 \mathrm{~mm}$. Measurements with a small thermocouple placed at various locations above the source cell showed the temperature of the carrier gas declined as it traveled up the nozzle, reaching $\sim 75^{\circ} \mathrm{C}$ by the position of the substrate. As shown in Fig. 
2(b), the largest temperature change occurred within the first $1-2 \mathrm{~cm}$ above the source cell. Despite this however, only a small amount of vapor was observed to condense on the inner walls of the nozzle or elsewhere in the chamber, with most undeposited material being captured instead inside a trap downstream from the outlet of the chamber. The sample was attached to the underside of a glass window on the lid of the chamber and maintained close to room temperature through thermal contact.

The pressure inside the chamber was fixed at 5 psi above ambient by a regulator. After exiting the chamber through a hole in the bottom, carrier gas was scrubbed of tetracene by successive filter cartridges and passed through an oxygen analyzer. The flow rate was controlled by a needle valve and monitored with a flowmeter. The gas velocity in the nozzle was calculated based on its diameter, the chamber pressure and temperature, and the volumetric flow rate out the end of the apparatus.

After the gas flow exited the nozzle it behaved like a semi-free jet, impinging on the substrate. For moderately large Reynolds numbers, but below the onset of instability ( $\operatorname{Re}<\sim 2000$ ), a circular jet injected into a stagnant fluid of similar density develops into a steady slender flow. ${ }^{33}$ As the jet entrains fluid from the surroundings it gradually expands and the centerline velocity decreases, eventually becoming unsteady. ${ }^{34,35}$ The Reynolds number for our apparatus based on the nozzle diameter was $R e=U d / \nu \approx 175$ under typical experimental conditions, where $U \approx 1.0 \mathrm{~m} \mathrm{~s}^{-1}$ is the flow velocity and $v$ is the gas kinematic viscosity. The jet was thus expected to remain laminar for distances $z^{*} / d>10$, which is longer than the nozzle-sample separation distance.

The substrate consisted of a $1 \mathrm{~cm} \times 1 \mathrm{~cm}$ indium-tin-oxide-coated (ITO) glass slide to which a $1.9 \pm 0.3 \mu \mathrm{m}$ thick layer of bis(2-ethylhexyl)sebecate (BES) was applied by spin coating. Samples were transparent, allowing film growth to be monitored in situ using polarized optical microscopy (Fig. 2(a)). BES was chosen because it fully wets both ITO and tetracene, it is a moderately good solvent for tetracene, and its low vapor pressure leads to slow evaporation. In addition, since tetracene film growth in a BES solvent layer was previously reported under vacuum conditions, ${ }^{18}$ a direct comparison reveals differences arising from the use of ambient pressure conditions.

Prior to deposition, the entire apparatus was repeatedly evacuated and refilled with Ar until the oxygen concentration in the exit flow was $50-100 \mathrm{ppm}$. As discussed below, a small amount of tetracene oxidation was observed to occur at this partial pressure of oxygen, which presumably could be reduced further if desired by the use of more highly purified carrier gas. The source cell was then heated to the desired temperature and allowed to equilibrate with the gas flow turned on. Deposition was prevented from occurring during the equilibration period by a shutter placed between the nozzle and the sample. The shutter was withdrawn to initiate deposition and film growth was recorded though a series of time-lapse micrographs.

After deposition samples were removed from the chamber for further analysis. The total amount of deposited tetracene was determined by dissolving films in hexane and measuring the concentration of the resulting solution by UVvis spectroscopy. ${ }^{36} \mathrm{X}$-ray diffraction measurements were performed on films that had been rinsed by briefly dipping in hexane; this removed most of the BES solvent while leaving the tetracene film largely undisturbed.

\section{RESULTS AND DISCUSSION}

\section{Hydrodynamics, deposition, and gas-phase aggregation}

The impinging jet of carrier gas can be modeled as twodimensional axisymmetric stagnation point flow, a classical problem in fluid mechanics characterized by a normally incident, radially-symmetric stream of fluid impinging on a surface. ${ }^{37}$ Because the solvent layer coating the substrate was very thin, we treat the flow as impinging on a flat, incompressible surface. Experiments showed that the deposition rates on bare and liquid-coated surfaces were indistinguishable, indicating that the presence of the liquid did not affect deposition. Thus, the sticking coefficients on bare and liquidcoated substrates were nearly the same.

Along the z-direction the flow field can be divided into two regions: (i) a diffusive boundary layer immediately adjacent to the substrate, and (ii) an outer flow region. In the outer flow region the streamlines are hyperbolic with the velocity components of the gas approximately given by ${ }^{38}$

$$
\begin{gathered}
v_{r}=\alpha r, \\
v_{z}=-2 \alpha z,
\end{gathered}
$$

where $\alpha$ is a constant which depends on the experimental geometry and flow velocity, and $r$ is the radial distance from the center of the impinging jet (Fig. 2(c)). Note from Eq. (1b) that the vertical component of the gas velocity is independent of radial position, and hence the amount of time required for a fluid parcel exiting the nozzle to reach a given height $z$ depends only on $z$, and not on its radial position. Therefore if vapor molecules aggregate in the gas phase, there should be no radial variation in the size of deposited particles. Since the Reynolds number under typical experimental conditions was small, the flow conditions are assumed to be laminar.

The transport of molecules or particles across the diffusive boundary layer is subject to several influences, including diffusive, thermophoretic, gravitational, and electrostatic forces. ${ }^{38-40}$ As shown below, there is evidence that the majority of particles reaching the liquid layer were less than $1 \mu \mathrm{m}$ in diameter. For such small particles, whose Stokes numbers $S t=2 \rho U r_{p}^{2} /(9 \mu R) \ll 1$, where $m, r_{p}$, and $\rho$ are the particle mass, radius, and density, respectively, and $R$ is the radius of the substrate, direct impaction due to particle momentum and gravitational settling are both negligible. ${ }^{41}$ The contribution of electrostatic forces was tested by comparing the deposition rates of tetracene onto bare ITO-coated glass slides that were electrically grounded, electrically isolated, or connected to $(+)$ or $(-)$ terminals of a $2 \mathrm{kV}$ DC power supply. The deposition rates in all cases were indistinguishable within 
experimental error, demonstrating that electrostatic forces did not significantly affect the deposition rate.

Because the temperature of the substrate was about $50{ }^{\circ} \mathrm{C}$ cooler than that of the impinging jet, the contribution of thermophoresis to deposition should be also considered. Thermophoretic motion results from gas molecules striking the warm side of a particle or molecule with a larger average momentum than those on the cold side, producing a net force acting in the direction of decreasing temperature. If the particle is small enough to neglect its own internal thermal gradient, the flux due to thermophoresis can be written as ${ }^{42}$

$$
J_{t}=\frac{-c_{o} K \nu}{T_{g}} \nabla T
$$

where $c_{o}$ is the tetracene concentration in the gas, $T_{g}$ is the temperature of the carrier gas, and $K$ is the particle thermophoretic coefficient. For small particles whose diffusive motion is in the free molecular regime, $K=0.55$. $^{43}$ The average temperature gradient is given by

$$
\nabla T=1.05 \operatorname{Pr} r^{0.36}\left(\frac{U}{d \nu}\right)^{1 / 2}\left(T_{s}-T_{g}\right)
$$

where the Prandtl number $\operatorname{Pr}=\nu / \kappa, \kappa$ is the gas diffusivity, and $T_{s}$ is the temperature of the substrate. Under typical experimental conditions the thermophoretic contribution to the total mass flux predicted by Eq. (2) is about an order of magnitude smaller than that due to convection/diffusion. Thus, although thermophoresis can be quite important for large temperature gradient flows, in the near-ambient conditions next to the substrate it is expected to be of only minor importance.

Limiting the analysis therefore to just the combined effects of convection/diffusion, the flux $J$ of molecules crossing the diffusive layer per unit area per unit time can be written as

$$
J \approx \frac{c_{o} B S c^{1 / 3} \operatorname{Re}^{1 / 2} D}{d}
$$

where the Schmidt number $S c=\nu / D$, and $D$ is the diffusion coefficient which we compute using Epstein's equation ${ }^{44}$

$$
D_{i}=\frac{3}{8 \rho r_{p}^{2} \delta} \sqrt{\frac{m k T_{g}}{2 \pi}}
$$

where $\delta=0.91$ is the accommodation coefficient. ${ }^{45}$ Equation (4) has been shown to provide a fairly accurate description of particle flux near the stagnation point under a wide range of experimental conditions. ${ }^{46}$ The constant $B$ in Eq. (4) depends on the experimental geometry and whether flow is laminar or turbulent. For the conditions used here (nonuniform laminar flow from a circular jet at a reduced nozzlesample distance $z^{*} / d=3.4$ ), a value $B=19.9$ is appropriate near the stagnation point. ${ }^{47}$ Values applicable to other geometries and flow conditions have been tabulated, for example, by Chin and Tsang. ${ }^{39}$

The deposition efficiency $\varepsilon=J / U c_{o}$, defined as the fraction of gas-phase tetracene deposited on the sample, was measured at a (radially averaged) jet velocity of $U=0.82 \mathrm{~m} \mathrm{~s}^{-1}$ by attaching a glass wool cartridge to the end of the nozzle and comparing the amount of tetracene captured in the cartridge to that deposited on a $1 \mathrm{~cm}^{2}$ sample under identical conditions without the cartridge. This gave $\varepsilon=10 \pm 5 \%$, which compares to the efficiency predicted by Eq. (4) of $8.6 \%$ for an aerosol composed entirely of tetracene monomers (i.e., if no gas-phase aggregation occurs). We note that these deposition efficiencies are higher than typically achieved in PVD or OMBE film growth, where omnidirectional fluence from the source cell results in only a small fraction of sublimate actually being deposited the substrate.

Because the temperature of the carrier gas was below the sublimation temperature of tetracene, it is possible that some aggregation took place as the vapor traveled from the source cell to the sample. It is well established from studies of soot formation in hydrocarbon flames that the aggregation of gas-phase polycyclic aromatic hydrocarbons under transport-limited conditions leads to aggregates with size distributions that exhibit dynamic scaling. ${ }^{48-53}$ Using this as a model for the aggregation of tetracene in OVLS deposition, the average particle size would be expected to increase as $\langle i\rangle$ $\sim t^{z}$, where $i$ is the number of molecules in the cluster and $t$ is the flight time. ${ }^{54}$ The exponent $z$ depends on the details of the aggregation kinetics but for many diffusion-limited cluster aggregation processes $z=1 .^{55}$ The particle radius scales with the number of molecules in the particle as $r_{p} \sim i^{x}$ where $x=1 / 3$ for compact particles. If we approximate the full size distribution with a $\delta$-function centered at $\langle i\rangle$ and make use of the fact that $t \sim U^{-1}$, Eqs. (4) and (5) can be combined and written in terms of the flow velocity as

$$
J=A U^{\beta}
$$

where $A$ is a constant and $\beta=(4 x z-z) / 3+1 / 2$.

Equation (6) predicts that in the absence of aggregation $\beta=0.5$. This was the situation in the experimental system employed in Ref. 30, where a heated flow tube was used to prevent aggregation in an experiment studying carrier gasassisted deposition of aluminum tris-(8 hydroxyquinoline) onto a solid film of $\mathrm{N}^{\prime}$-diphenyl-N, $\mathrm{N}^{\prime}$-bis(3-methylphenyl)1$1^{\prime}$ biphenyl-4-4'diamine and the value predicted here of $\beta=0.5$ was observed. Conversely, in the case of aggregation the expected value is closer to $\beta \approx 0.83$. Figure 3 shows a log-log plot of the deposition rate versus flow velocity measured over a $1 \mathrm{~cm}^{2}$ area centered above the nozzle. The deposition rate obeys the expected power-law dependence on flow velocity with a slope $\beta=0.89 \pm 0.08$. This is significantly larger than the value 0.5 observed in the absence of aggregation, and agrees with the simple aggregation model described above to within experimental uncertainty. These observations suggest that some clustering of gas-phase tetracene probably occurs during transport to the substrate.

Fully developed pipe flow generally occurs when the length of the nozzle exceeds $L>0.057 d R e \approx 3.4 \mathrm{~cm} .{ }^{56}$ Since $L=7 \mathrm{~cm}$ in the experimental apparatus, the radial velocity profile should therefore more closely resemble fully developed (Poiseuille) flow than uniform flow. The partial pressure of tetracene, and hence the flux of tetracene to the sample 


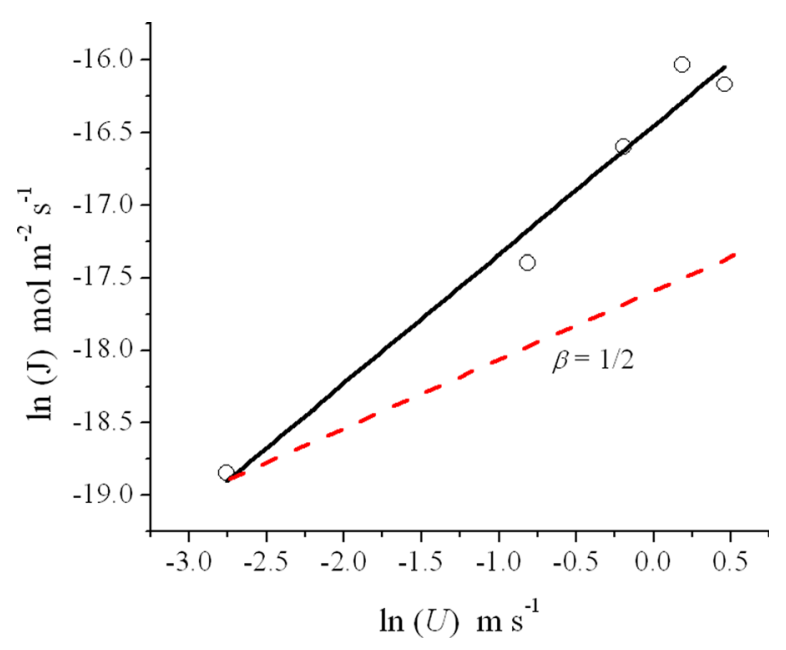

FIG. 3. The deposition rate, $j$ increased with flow velocity as $j \sim U^{B}$, with $\beta=0.89$ (solid line). In the absence of aggregation, the predicted exponent is $\beta=0.5$ (dashed line).

surface, should therefore be largest near the stagnation point. $^{57,58}$ Measurements of film thickness performed by small-spot UV-vis spectroscopy on a sample deposited onto bare ITO/glass showed that this was indeed the case (Fig. 4). The open points show the relative optical density at $530 \mathrm{~nm}$ (the peak absorbance wavelength for tetracene) as a function of radial position measured with a spot size of about $1 \mathrm{~mm}$. The solid line provides a comparison to the normalized mass transfer rate predicted for fully developed flow, based on the approximate solutions for an impinging circular jet given by Scholtz and Trass under conditions similar to those used in the experiment. ${ }^{59}$ The observed film thickness profile can be seen to be close to, but slightly broader than what would be expected for fully developed flow. One consequence of the radial variation in the flux was that crystals that formed far from the stagnation point (at radial distances $\geq \sim 5 \mathrm{~mm}$ ) tended to be larger and more widely separated than those formed near the center. Note that it should be possible to obtain other radial profiles by changing the nozzle shape, experimental

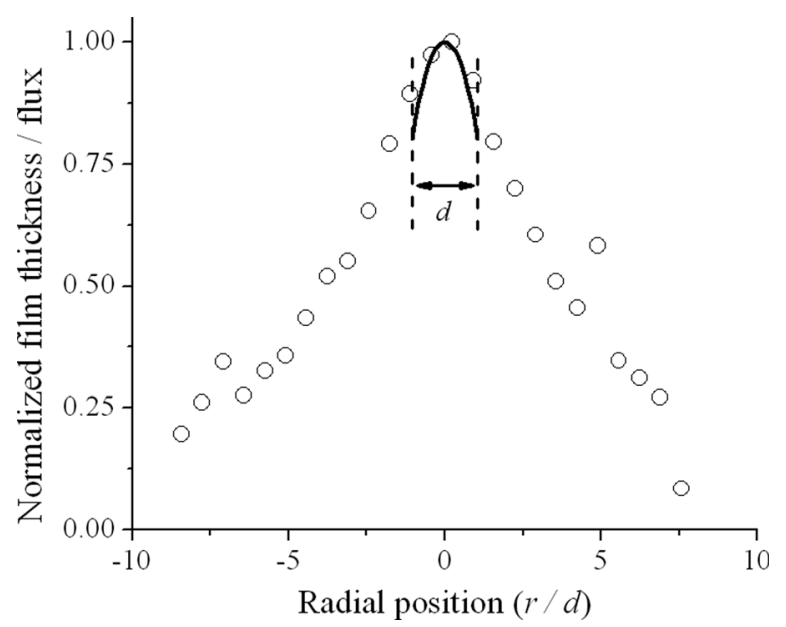

FIG. 4. Dependence of deposition rate on radial position determined by small spot UV-VIS absorbance spectroscopy for a sample deposited onto bare ITO/glass. The diameter of the nozzle is shown with dashed lines. Solid line is the predicted flux for fully developed flow. geometry or Reynolds number to produce, for example, more uniform or more sharply peaked deposition. ${ }^{60}$

\section{Film morphology and growth kinetics}

We turn now to a description of some of the features of the films deposited using this method. Figure 5 presents a sequence of optical micrographs showing crystal nucleation and film growth for a representative sample. The images were acquired in situ during tetracene deposition onto a BEScoated ITO/glass slide and show a $1 \mathrm{~mm}^{2}$ region near the stagnation point. Within this area the radial variation in the flux was less than $10 \%$ across the field of view. The first crystals became visible several minutes after the start of deposition (Fig. 5(a)), rapidly increasing in number over the next 1-2 min (Fig. 5(b)). Thereafter new crystal formation effectively ceased, while existing crystals continued to grow (Fig. $5(\mathrm{c})$ ). Eventually crystals became large enough to overlap, forming an interconnected semicontinuous film (Fig. 5(d)).

The principle stages of film formation represented in Fig. 5 are more clearly distinguished in a plot of crystal number density versus deposition time, shown in Fig. 6. The data in Fig. 6 were determined by counting the number of crystals in each frame of a sequence of optical micrographs starting at the onset of deposition, spaced in $30 \mathrm{~s}$. intervals. Three distinct regimes can be identified: (1) an induction regime, prior to the onset of crystal formation; (2) a nucleation regime during which essentially all crystals were formed; (3) a growth regime, during which crystals increased in size but no new crystals formed. We note the findings in Fig. 6, which are the first reported description of time-dependent nucleation in an OVLS film, suggest there are qualitative differences from the nucleation kinetics usually observed during island formation and film growth using PVD or OMBE. $^{62}$ In particular, the induction regime-a common
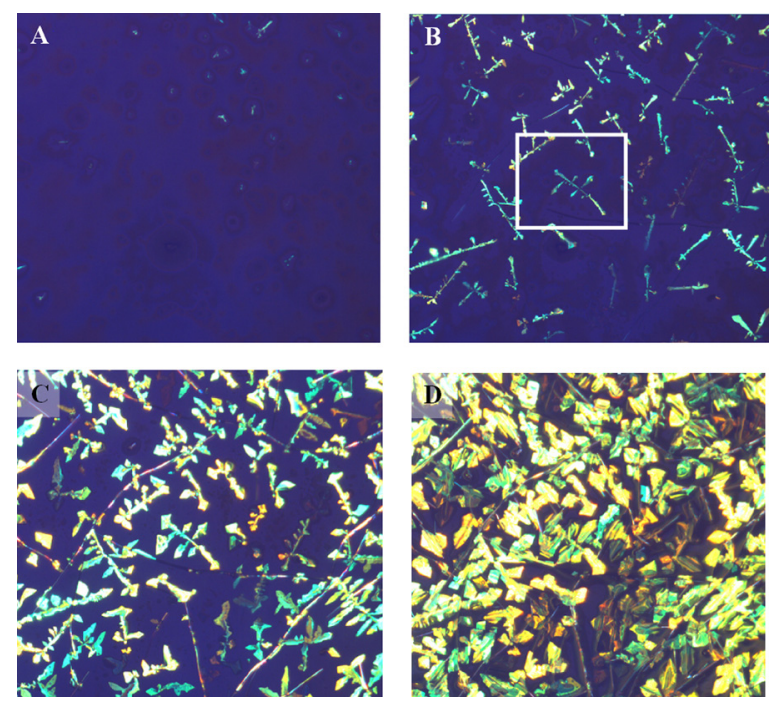

FIG. 5. Polarized optical micrographs showing development of a representative film during deposition. Time after the start of deposition: (a) $4.4 \mathrm{~min}$, (b) $15.5 \mathrm{~min}$, (c) $75.5 \mathrm{~min}$, (d) $8 \mathrm{~h} 51 \mathrm{~min}$. Images measure $1 \mathrm{~mm} \times 1 \mathrm{~mm}$. Variations in the color and brightness of different crystals resulted from their having different thicknesses and azimuthal orientations with respect to the polarization axes of the optical microscope. The development of the crystal highlighted by the box in part (b) is shown in Fig. 8. 


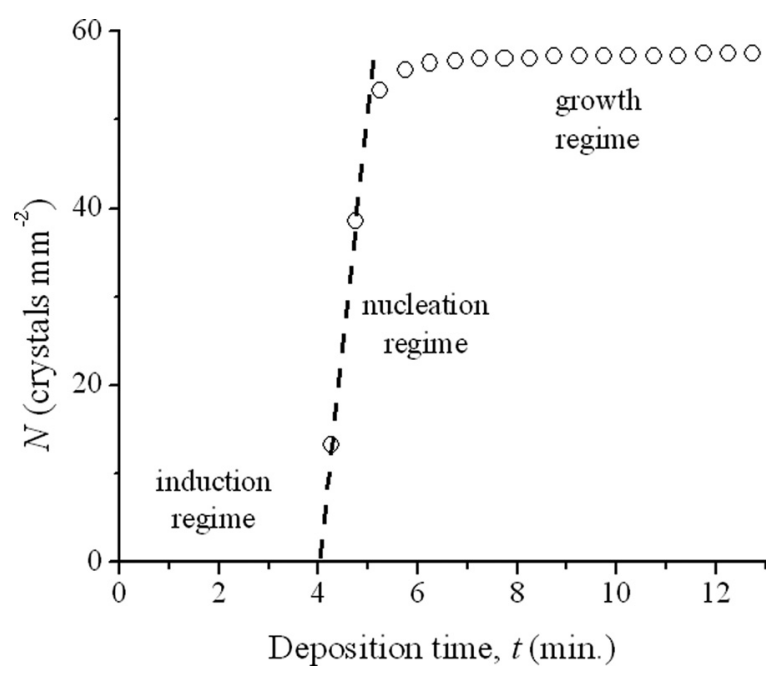

FIG. 6. Crystal formation and growth took place in three distinct stages: an induction regime prior to crystal formation; a nucleation regime typified by a large rate of new crystal formation; and a growth regime during which existing crystals continued to grow but almost no new crystals formed.

feature in liquid-phase crystallization-is usually nonexistent for growth on bare surfaces, and nucleation continues at a reduced rate well into the growth regime. The induction regime in OVLS deposition can be understood in terms of the concentration of tetracene in the solvent layer, which steadily increases until eventually reaching a sufficiently high supersaturation $C \geq C^{*}$ to initiate nucleation. For the sample in Fig. 6 this occurred about 4 min after the start of deposition. Based on the flux rate $\left(J=10^{16}\right.$ molecules $\left.\mathrm{m}^{-2} \mathrm{~s}^{-1}\right)$ and the solvent layer thickness $(d=1.9 \mu \mathrm{m})$, the critical supersaturation concentration can be estimated to be $C^{*}=4 \pm 2 \mathrm{mM}$. This compares to the saturation concentration of tetracene in BES which was separately measured to be $0.7 \pm 0.1 \mathrm{mM}$.

Also shown in Fig. 6, the formation of new crystals was limited to a brief period of time (the nucleation regime) after which all nucleation effectively ceased. This indicates either a heterogeneous nucleation mechanism involving a finite number of seeding sites, such as impurities, which eventually become titrated, or an eventual reduction in the solution phase concentration of tetracene to $C<C^{*}$ as a result of uptake by growing crystals. The former is inconsistent with our observation that the nucleation density generally tended to increase with increasing deposition rate, suggesting the latter is more likely. The lack of new crystal nucleation in the growth regime also shows that the flux of vapor-phase tetracene reaching the solvent layer contained no aerosol particles large enough to serve as critical nuclei. This indicates that, despite the use of near ambient conditions, vapor-phase aggregates must be small. We note that under typical experimental conditions the arrival of tetracene from the vapor was never directly observed by optical microscopy, even at the highest magnification used $(500 \times)$. This means that the largest gas-phase clusters reaching the surface must be less than about $1 \mu \mathrm{m}$ in size.

Although the in-plane orientation of crystals in BES appeared to be random, the out-of-plane (polar orientation) was almost perfectly uniform, as can be inferred from the

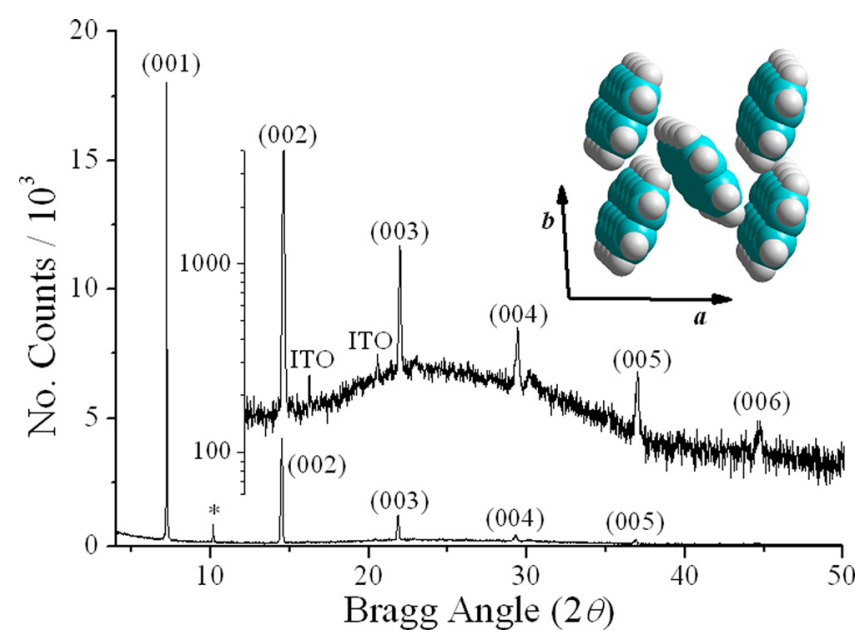

FIG. 7. X-ray diffractogram of tetracene deposited onto BES-coated ITO/ glass after rinsing in hexane to remove excess solvent. Note reflections exclusively from the (001) planes. The peak denoted with an asterisk is assigned to 5,12-napthacenequinone, an oxidation impurity of tetracene. Inset shows the unit cell viewed looking down on the substrate.

X-ray diffractogram in Fig. 7. The data in Fig. 7 were acquired from a $\sim 75 \mathrm{~nm}$ thick film that had been rinsed in hexane to remove most BES. The peak positions agree with the previously reported bulk crystal structure of tetracene, ${ }^{61}$ with all major reflections corresponding to the $(00 l)$ family of planes. This indicates that the $a b$-plane of the unit cell was very nearly coplanar with the substrate. A small impurity peak at $2 \theta=10^{\circ}$ is assigned to 5,12-napthacenequinone, the major oxidation product of tetracene. The same impurity was also attributed to the appearance of needle-shaped crystals (not shown) also observed on most samples. X-ray diffraction measurements on films deposited onto bare surfaces (without a solvent layer) were identical to the results in Fig. 7, indicating that the preferred planar orientation and crystal structure of tetracene were not affected by the BES solvent layer.

To illustrate some further aspects of the growth kinetics, the growth of one representative crystal is followed in more detail in Fig. 8. Nucleation occurred near the point indicated by an arrow in the second panel. The primary axis of the crystal developed first, with branches appearing soon afterwards. Measurements of the branching angles from a number of crystals showed that the majority made an angle of $90 \pm 5^{\circ}$ with the primary axis, consistent with growth along the [100] and [010] axes, which form an angle of $93.7^{\circ}$ (see inset, Fig. 7). ${ }^{61}$ This contrasts with the observations reported in Ref. 18 for tetracene growth in BES under vacuum conditions, where branching angles of approximately $35^{\circ}-40^{\circ}$ were predominately observed, consistent with growth along the $[110]$ and $[1 \overline{1} 0]$ directions.

As shown in Fig. 8, once crystals reached a certain size longitudinal growth slowed and the branch tips began to coarsen, forming prismatic shapes with well-defined facets. Coarsening progressed from branch extremities inwards, with the primary crystal axis growing most slowly. These characteristics are consistent with a diffusion-driven mechanism in which crystals receive new growth units primarily from the surrounding quasi-2D concentration field, but also including growth units able to penetrate the crevices between 

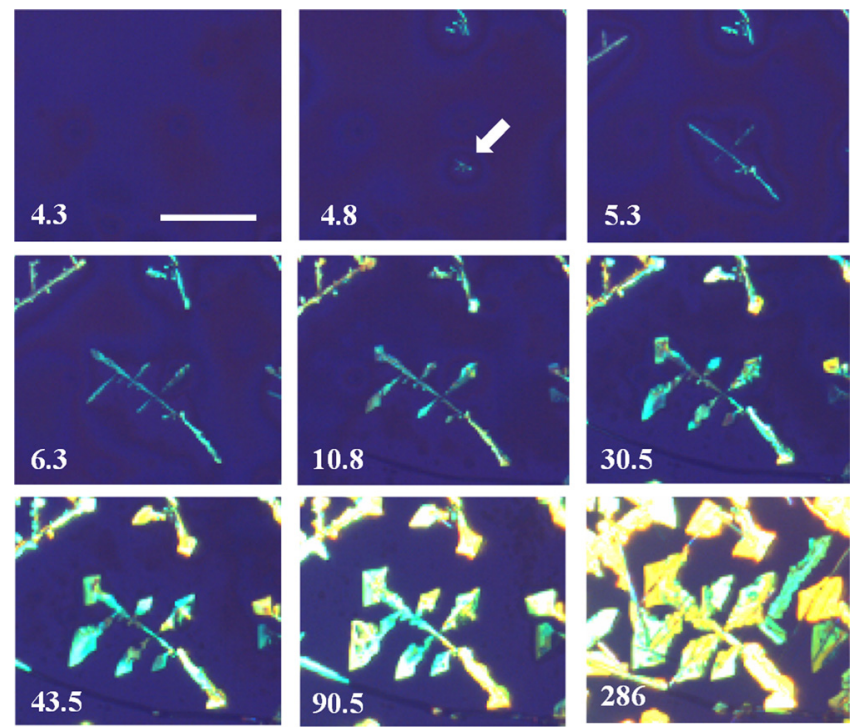

FIG. 8. Polarized optical micrographs showing development of a representative crystal. Time after the start of deposition in minutes is indicated in the lower left of each panel. Scale bar is $100 \mu \mathrm{m}$.

branches by arriving atop the area proscribed by existing crystals. Qualitatively similar morphological progressions are frequently observed in OMBE, where initially branched crystals coarsen inward from the extremities as the coverage is increased. Aside from the difference in branching angles mentioned above, in most other respects the findings observed here for ambient pressure-deposited films were very similar to those reported for tetracene films grown in BES under vacuum conditions, showing that the impinging jet technique produces similar results under more technologically favorable conditions.

To compare these results to films prepared without a solvent, Fig. 9 shows an electron micrograph of a tetracene film grown on bare ITO/glass (i.e., with no solvent layer) under conditions otherwise similar to those used to prepare the OVLS-deposited films discussed above. Without the solvent layer crystals were typically 1-2 orders of magnitude smaller in size and nucleation densities 1-2 orders of magnitude larger. Growth habits also tended to be more compact. X-ray

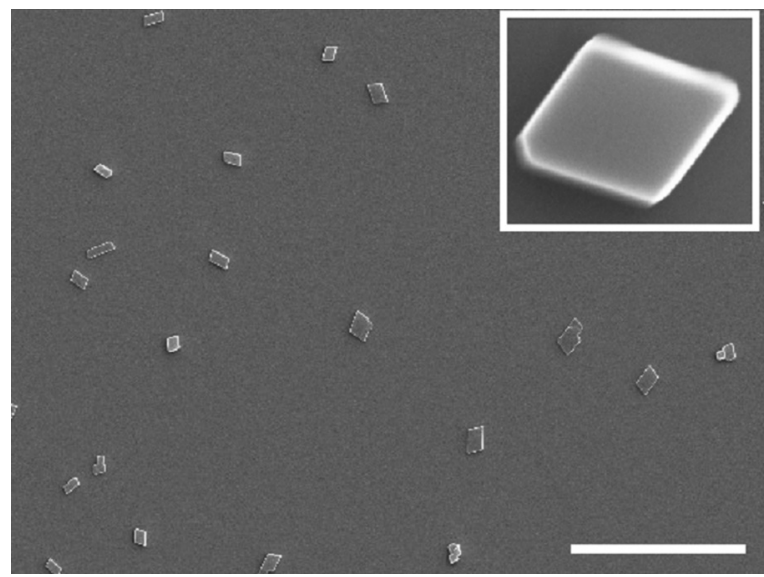

FIG. 9. Scanning electron micrograph of a tetracene film deposited onto bare ITO/glass (i.e., with no solvent layer). Scale bar is $20 \mu \mathrm{m}$. Inset shows a representative crystal at higher magnification $(3 \times 2.5 \mu \mathrm{m})$. diffraction measurements on such films gave results that were essentially identical to those for films deposited with a solvent layer, described above.

The most important kinetic parameter affecting the structure of vapor-deposited thin films is the ratio of the surface diffusion and flux rates, $D / F .^{62}$ Large values of $D / F$ normally lead to small nucleation densities and low degrees of crystal branching, although these properties are also affected by the size of the critical nucleus and other kinetic parameters. Comparing OVLS-grown films to those deposited onto bare ITO substrates under similar conditions, it is therefore surprising to observe that the former had lower nucleation densities but also crystal habits that at low coverage were significantly more branched. The first characteristic seems to imply a larger ratio $D / F$, whereas the latter implies a smaller one. We postulate this behavior may be explained in terms of a larger critical nucleus size in the liquid solvent compared to the bare surface. Referring to Fig. 5, in order for nucleation to occur in the liquid a supersaturated state is required, a state that develops as the solution concentration builds up during the induction regime. Once the critical concentration has been reached, new crystal formation occurs rapidly and the nucleated seeds receive a very high effective flux of growth units from the combined sources of the incoming vapor and excess dissolved tetracene in the supersaturated solution. This leads to the initially branched forms displayed by crystals in the early stages of OVLS growth. Once the solution has been depleted of most of the excess by the incorporation of solute molecules into growing seeds, neighboring crystals begin to compete for growth units, growth slows and the habits begin to become more compact. Although speculative, this picture could explain many of the differences observed between OVLS and dry substrate films. These and additional details of the growth kinetics will be explored further in a future publication. ${ }^{63}$

Finally, we note that the findings reported here for tetracene were broadly similar to those observed for several other tested compounds, including anthracene, rubrene, and $\alpha$-sexithiophene (6T). A representative example is presented in Fig. 10 , which shows a film of $6 \mathrm{~T}$ deposited into a BES solvent layer on ITO/glass at a source cell temperature of $185^{\circ} \mathrm{C}$ and flow velocity of $4.1 \mathrm{~m} \mathrm{~s}^{-1}$. The nucleation kinetics were similar to those of tetracene, with distinct induction, nucleation,

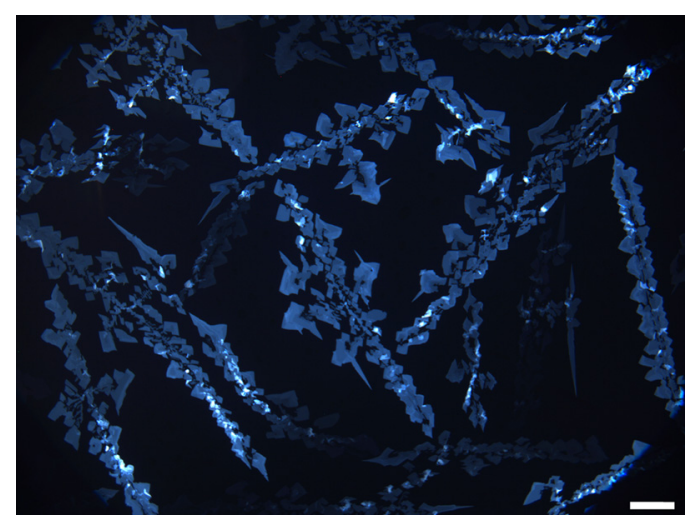

FIG. 10. Polarized optical micrograph of a $6 \mathrm{~T}$ film deposited onto BEScoated ITO/glass. Scale bar is $100 \mu \mathrm{m}$. 
and growth regimes. Also similar to tetracene, $6 \mathrm{~T}$ deposited onto BES-coated substrates resulted in much larger crystals (up to $\sim 1 \mathrm{~mm}$ in size) than on bare surfaces, and crystals displayed similar planar, branched growth habits. These observations suggest that the findings reported above for tetracene should be applicable to a range of other OMC-forming compounds.

\section{CONCLUSIONS}

A method for comparatively rapid and mass-efficient deposition of highly crystalline organic films under near ambient conditions of pressure and temperature has been reported. By delivering an organic precursor via an impinging gas jet to a solvent-coated substrate, transport across the diffusive boundary layer near the substrate is enhanced, permitting flux rates comparable to those achieved in vacuum and producing very similar crystalline films. Although there is evidence for some solid aerosol formation during vapor delivery, gas phase clusters appear to be small enough to dissolve in the solvent layer. Combining gas jet delivery with OVLS growth leads to larger crystals and lower nucleation densities than on bare surfaces, with nucleation and growth kinetics that reflect characteristics of both surface and liquidphase growth. Preliminary results from related compounds suggest that these findings should be broadly applicable to a range of organic materials.

\section{ACKNOWLEDGMENTS}

This work was supported by the National Science Foundation (DMR-0705908). The authors thank C. Lund and B. Ricker for their contributions.

${ }^{1}$ G. R. Desiraju, Crystal Engineering - The Design of Organic Solids (Elsevier, New York, 1989); M. Pope and C. E. Swenberg, Electronic Properties in Organic Crystals and Polymers, 2nd ed. (Oxford University Press, Oxford, 1999).

${ }^{2}$ R. F. Service, Science 278, 383 (1997); F. Garnier, R. Hajlaoui, A. Yasser, and P. Srivastava, Science 265, 1684 (1994); C. J. Drury, C. M. J. Mutsaers, C. M. Hart, M. Matters, and D. W. de Leeuw, Appl. Phys. Lett. 73, 108 (1998).

${ }^{3}$ M. C. Petty, Biosens. Bioelectron. 10, 129 (1995).

${ }^{4}$ N. Greenham and R. H. Friend, Solid State Physics: Advances in Research and Applications, 49, edited by H. Ehrenreich and F. Spaepen (Academic, San Diego, 1995), pp. 1-149.

${ }^{5}$ A. J. Lovinger and L. J. Rothberg, J. Mater. Res. 11, 1581 (1996); H. E. Katz, J. Mater. Chem. 7, 369 (1997); F. Garnier, Chem. Phys. 227, 253 (1998); G. Horowitz, Adv. Mater. 10, 365 (1998).

${ }^{6}$ J. Singleton and C. Mielke, Contemp. Phys. 43, 63 (2002).

${ }^{7}$ J. S. Miller and A. J. Epstein, Angew. Chem. Int. Ed. 33, 385 (1994).

${ }^{8}$ M. Mas-Torrent, P. Hadley, S. T. Bromley, X. Ribas, J. Tarres, M. Mas, E. Molins, J. Veciana, and C. Rovira, J. Am. Chem. Soc. 126, 8546 (2004).

${ }^{9}$ J. H. Schön, C. Kloc, R. A. Laudise, and B. Batlogg, Phys. Rev. B 58, 12952 (2002).

${ }^{10}$ R. W. I. de Boer, T. M. Klapwijk, and A. F. Morpurgo, Appl. Phys. Lett. 83, 4345 (2003).

${ }^{11}$ D. J. Gundlach, J. A. Nichols, L. Zhou, and T. N. Jackson, Appl. Phys. Lett. 80, 2925 (2002).

${ }^{12}$ Crystal and Solid State Physics, edited by O. Madelung (Springer-Verlag, New York, 1985).

${ }^{13}$ J. G. Laquindanum, H. E. Katz, A. J. Lovinger, and A. Dodabalapur, Chem. Mater. 8, 2542 (1996); T. Minakata, H. Imai, and M. Ozaki, J. Appl. Phys. 72, 5220 (1992); A. J. Lovinger, H. E. Katz, and A. Dodabalapur, Chem. Mater. 10, 3275 (1998); A. J. Makinen, A. R. Melnyk, S. Schoemann, R. L. Headrick, and Y. Gao, Phys. Rev. B 60, 14683 (1999).
${ }^{14}$ E. A. Silinsh and V. Ėápek, Organic Molecular Crystals Interaction, Localization, and Transport Phenomena (AIP, New York, 1994).

${ }^{15} \mathrm{~N}$. Karl, in Crystals - Growth, Properties and Applications, edited by H. C. Freyhard (Springer, New York, 2001).

${ }^{16}$ A. Afzali, C. D. Dimitrakopoulos, and T. L. Breen, J. Am. Chem. Soc. 124, 8812 (2002).

${ }^{17}$ S. R. Forrest, Chem. Rev. 97, 1793 (1997).

${ }^{18}$ M. Voigt, S. Dorsfeld, A. Volz, and M. Sokolowski, Phys. Rev. Lett. 91, 026103-1 (2003).

${ }^{19}$ R. S. Wagner and W. C. Ellis, Appl. Phys. Lett. 4, 89 (1964).

${ }^{20}$ R. S. Wagner, Whisker Technology, Chap. 3, edited by A. P. Levitt (Wiley, New York, 1970).

${ }^{21}$ T. J. Trentler, K. M. Hickman, S. C. Goel, A. M. Viano, P. C. Gibbons, and W. E. Buhro, Science 270, 1791 (1995).

${ }^{22}$ A. M. Morales and C. M. Lieber, Science 279, 208 (1998).

${ }^{23}$ X. Liu and M. Wuttig, Phys. Rev. B 73, 033405-1 (2006).

${ }^{24}$ T. Shimada, Y. Ishii, K. Ueno, and T. Hasegawa, J. Cryst. Growth 311, 163 (2008).

${ }^{25}$ Y. Ishii, T. Shimada, N. Okazaki, and T. Hasegawa, Langmuir 23, 6864 (2007).

${ }^{26}$ Y. Takeyama, S. Maruyama, and Y. Matsumoto, Cryst. Gr. Design 11, 2273 (2011).

${ }^{27}$ F. S. Wilkinson, R. F. Norwood, J. M. McLellan, L. R. Lawson, and D. L. Patrick, J. Am. Chem. Soc. 128, 16468 (2006).

${ }^{28}$ R. R. Lunt, J. B. Benziger, and S. R. Forrest, Adv. Mat. 22, 1233 (2010).

${ }^{29}$ S. S. Lee, C. S. Kim, E. D. Gomez, B. Purushothaman, M. F. Toney, C. Wang, A. Hexemer, J. E. Anthony, and Y.-L. Loo, Adv. Mat. 21, 3605 (2009).

${ }^{30}$ M. A. Baldo, M. Deutsch, P. E. Burrows, H. Gossenberger, M. Gerstenberg, V. S. Ban, and S. R. Forrest, Adv. Mater. 10, 1505 (2009).

${ }^{31}$ M. Shtein, H. F. Gossenberger, J. B. Benziger, and S. R. Forrest, J. Appl. Phys. 89, 1470 (2001).

${ }^{32}$ M. A. Baldo, V. G. Kozlov, P. E. Burrows, S. R. Forrest, V. S. Ban, B. Koene, and M. E. Thompson, Appl. Phys. Lett. 71, 3033 (1997).

${ }^{33}$ S. J. Kwon and I. W. Seo, Exp. Fluids 38, 801 (2005).

${ }^{34}$ A. J. Reynolds, J. Fluid Mech. 14, 552 (1962).

${ }^{35} \mathrm{H}$. Schlichting, Z. Angew. Math. Mech. 13, 260 (1933).

${ }^{36}$ The extinction coefficient of tetracene in mixed hexanes was determined to be $321,000 \mathrm{~cm}^{-1} \mathrm{M}^{-1}$ at $274 \mathrm{~nm}$.

${ }^{37}$ H. Schlichting, Boundary Layer Theory (McGraw-Hill, New York, 1960).

${ }^{38}$ D. F. Rogers, Laminar Flow Analysis (Cambridge University Press, Cambridge, 1992).

${ }^{39}$ D.-T. Chin and C.-H. Tsang, J. Electrochem. Soc.: Electrochem. Sci. Technol. 125, 1461 (1978).

${ }^{40}$ D. W. Cooper, M. H. Peters, and R. J. Miller, Aerosol. Sci. \& Technol. 11, 133 (1989).

${ }^{41}$ M. H. Peters, D. W. Cooper, and R. J. Miller, J. Aerosol. Sci. 20, 123 (1989).

${ }^{42}$ G.-W. Bae, C. S. Lee, and S. O. Park, Aerosol. Sci. Technol. 23, 231 (1995).

${ }^{43}$ L. Z. Waldmann and K. H. Schmitt, Aerosol Science, edited by C. N. Davies (Academic, London, 1966).

${ }^{44}$ J. Cai and V. M. Sorensen, Phys. Rev. E 50, 3397 (1994).

${ }^{45}$ K. J. Duan, G. J. Smallwood, and F. Liu, J. Heat Transf. 130, 121201-1 (2008).

${ }^{46}$ See for example: Y. Otani, H. Emi, C. Kanaoka, and K. Kato, J. Aerosol Sci. 20, 787 (1989); B. Y. H. Liu and K. Ahn, Aersol. Sci. Technol. 6, 215 (1987).

${ }^{47}$ The parameter B was calculated from Equation III-1 given in Ref. 39, which is based on a least-squares curve fitting to the theoretical predictions in M. T. Scholtz and O. Trass, AlChE J. 16, 82 (1970).

${ }^{48}$ W. Kim, C. M. Sorensen, D. Fry, and A. Chakrabarti, Aerosol Sci. 37, 386 (2006).

${ }^{49}$ K. A. Jensen, J. M. Suo-Antilla, and L. G. Blevins, Combust. Sci. Technol. 179, 2453 (2007).

${ }^{50}$ U. O. Köylü, G. M. Faeth, T. L. Farias, and M. G. Carvalho, Combust. Flame 100, 621 (1995).

${ }^{51}$ R. K. Chakrabarty, H. Moosmüller, W. P. Arnott, M. A. Garro, G. Tian, J. G. Slowik, E. S. Cross, J.-H. Han, P. Davidovits, T. B. Onasch, and D. R. Worsnop, Phys. Rev. Lett. 102, 235504 (2009) and references therein.

${ }^{52} \mathrm{X}$. Li and B. E. Logan, Environ. Sci. Technol. 31, 1237 (1997).

${ }^{53}$ D. A. Weitz and M. Y. Lin, Phys. Rev. Lett. 57, 2037 (1986).

${ }^{54}$ G. Sposito, Coll. Surf. A: Phys. Eng. Aspects 120, 101 (1997).

${ }^{55}$ M. H. Lin, H. M. Lindsay, D. A. Weitz, R. C. Ball, R. Klein, and P. Meakin, Proc. R. Soc. Lond. A 423, 71 (1989). 
${ }^{56}$ H. L. Langhaar, J. Appl. Mech. 9, 55 (1942).

${ }^{57}$ H. Martin, Adv. Heat Transfer 13, 1 (1977).

${ }^{58}$ F. Giralt, C.-J. Chia, and O. Trass, Ind. Eng. Chem., Fundam. 16, 21 (1977).

${ }^{59}$ M. T. Scholtz and Trass, AlChE J. 16, 90 (1970). Equation 23 along with values from Table I for $\mathrm{H} / \mathrm{R}=1$ was used in the calculation of the profile for fully developed flow. The calculation was performed using $\mathrm{Sc}=1.98$ and $\mathrm{Re}=216$ with the resulting Sherwood number normalized to the radial position $\mathrm{x} / \mathrm{R}=0$ for comparison to the experimental data.
${ }^{60}$ D. J. Phares, G. R. Smedley, and R. C. Flagan, Phys. Fluids 12, 2046 (2000).

${ }^{61}$ J. M. Robertson, V. C. Sinclair, and J. Trotter, Acta Cryst. 14, 697 (1961); R. B. Campbell, J. M. Robertson, and J. Trotter, Acta Cryst. 15, 289 (1962).

${ }^{62}$ J. W. Evans, P. A. Thiel, and M. C. Bartelt, Surf. Sci. Rep. 61, 1 (2006).

${ }^{63}$ A. Baronov, D. Shaw, K. Bufkin, C. Lund, B. L. Johnson, and D. L. Patrick (to be submitted). 